\title{
Audit on Practices of Stress Ulcer Prophylaxis in Intensive Care Unit Patients
}

\author{
Divya Gupta ${ }^{1}$, Anju R Bhalotra², Rahil Singh ${ }^{3}$
}

\begin{abstract}
Background: This audit was aimed at studying current practices regarding the use of stress ulcer prophylaxis (SUP) in the Indian critical care setup, with the background aim of raising awareness regarding the use and indications of SUP in critically ill patients.

Materials and methods: After registering the trial with the clinical trial registry, India, a structured audit questionnaire containing 26 questions pertaining to SUP was distributed through electronic media among clinicians working in the intensive care units (ICUs) across India. Responses obtained were statistically analyzed.

Results: The questionnaire was sent to 550 clinicians. Only 197 responded, of whom $91.4 \%$ were anesthesiologists, $5.6 \%$ were physicians, and $3 \%$ were intensivists. The audit revealed that 33\% respondents were unaware of the existing SUP guidelines and around $32 \%$ did not have protocols for SUP in their ICU. Sixty-nine percent of respondents felt that all ICU patients must receive SUP and $44.7 \%$ opined that it should be started on ICU arrival. Almost $94 \%$ knew that early enteral feeding is protective against stress ulceration. Only $24.9 \%$ responders agreed that there must be clear indications for SUP, and most of them were unaware of all the potential side effects. Once initiated, $43.7 \%$ respondents would stop prophylaxis when no indication was left, whereas 69 respondents would stop on ICU discharge.

Conclusion: There is a lack of awareness regarding initiation, choice of agent, adverse effects as well as termination of SUP in ICU and guidelines for the same. Institutional protocols should be in place and steps need to be taken to prevent unwarranted use.

Keywords: Critical care, $\mathrm{H} 2$ receptor locker, Proton pump inhibitor, Stress ulcer bleeding, Sucralfate.

Indian Journal of Critical Care Medicine (2020): 10.5005/jp-journals-10071-23368
\end{abstract}

\section{InTRODUCTION}

Stress ulcer prophylaxis (SUP) has been extensively used in critically ill patients to prevent upper gastrointestinal (GI) bleeding. However, a large number of patients in intensive care units (ICUs) continue to receive acid-suppressing medications for SUP; and this widespread overzealous use of drugs for prevention of stress ulcers in ICU patients is now an outdated practice. Commonly used acid-suppressing medications include proton pump inhibitors (PPIs) and histamine 2-receptor antagonists (H2RAs). Over time, these drugs have been studied in detail and compared with each other for the short- as well as long-term benefits and adverse effects, and the product labeling on acid suppressing medications has been changed to reflect the increased risks associated with their use. These include a wide spectrum of side effects such as longbone fractures, Clostridium difficile-associated diarrhea, electrolyte and vitamin deficiencies, drug interactions, thrombocytopenia, pneumonia, etc. Furthermore, some studies found no difference in $\mathrm{Gl}$ bleeding despite the use of acid-suppressing medication. The first and the last guideline regarding the use of SUP in the ICU was published in 1999 by the American Society of Health-system Pharmacists (ASHP). ${ }^{1}$ Since then, there has been no further update. No clear recent guidelines are available regarding the use of SUP in critically ill ICU patients. We therefore conducted this survey to assess the knowledge and awareness of the risks, benefits, and indications for SUP in ICU patients.

\section{Materials and Methods}

After taking approval from the institutional ethics committee and registering the trial with the clinical trial registry, India (CTRI/2019/03/017903), this prospective, cross-sectional survey was
${ }^{1-3}$ Department of Anaesthesiology and Critical Care, Maulana Azad Medical College, New Delhi, India

Corresponding Author: Divya Gupta, Department of Anaesthesiology and Critical Care, Maulana Azad Medical College, New Delhi, India, Phone: +91-99909-25895, e-mail: darydivs@yahoo.com

How to cite this article: Gupta D, Bhalotra AR, Singh R. Audit on Practices of Stress Ulcer Prophylaxis in Intensive Care Unit Patients. Indian J Crit Care Med 2020;24(3):160-167.

Source of support: Nil

Conflict of interest: None

conducted to study practices related to SUP prophylaxis in critical ICU patients across various hospitals in India. A well-structured audit questionnaire was prepared on Google docs, containing 5 questions regarding the experience and place of practice of the respondents and 21 questions pertaining to the use of SUP in ICU patents.

The questions mainly focused on indications and timing of initiation, titration, and discontinuation of various available alternatives. It was distributed among clinicians working in various ICUs across India through electronic media, and their voluntary participation was sought. Study objectives were clearly mentioned in the mail and anonymity of the respondent's identity as well as their responses was ensured. The first question to the participating clinicians was to know whether they were physicians, anesthesiologists, or intensivists. The next four questions were regarding the state and facility at which they worked, along with their experience in the ICU. The core set of 21 questions involved questions related to knowledge regarding the existence of any guidelines; whether any standard protocol was being in their 
setup; and types of drugs used for SUP, their indications and adverse effects, role of enteral feeding, and the appropriate time for discontinuation of the drug (Annexure 1). A reminder mail was sent twice at an interval of 1 week and the responses were collected and analyzed.

\section{RESULTS}

The questionnaire was sent to 550 clinicians through electronic media, of which 197 (35.81\%) were returned. Among them, 91.4\% was anesthesiologists, $5.6 \%$ physicians, and $3 \%$ intensivists. The majority of the respondents were from government teaching hospitals, $22.8 \%$ was from private nonteaching institutes and $3 \%$ from private nursing homes. Two third of the respondents was currently working in Delhi and three fourth of all respondents was from metro cities. Of the 197 respondents, 39 (19.8\%) had more than 10 years of experience in their respective fields, 15 (7.6\%) had 6-10 years of experience, 72 (36.5\%) had 3-6 years of experience, and 71 $(36 \%)$ had less than 3 years of experience. Of the respondents, 33 (16.8\%) had more than 5 years of experience in the ICU, 57 (28.9\%) had $1-5$ years, 49 (24.9\%) has 6-12 months, and 58 (29.4\%) had less than 6 months' experience in the ICU.

One hundred and thirty-two (67\%) respondents were aware of the guidelines regarding SUP and $68 \%$ said that SUP protocols were being followed in their respective ICUs. Sixty-nine percent respondents felt that all patients in the ICU should receive SUP prophylaxis, whereas 61 (31\%) were of the opinion that all patients should not routinely receive SUP prophylaxis (Fig. 1). Only 64 respondents (32.5\%) were aware that bleeding from stress ulceration is extremely uncommon in ICU patients, whereas $67.5 \%$ thought that it is common. As many as 184 (93.9\%) agreed that early enteral tube feeding (initiated within 48 hours of ICU admission) may be protective against stress ulceration, whereas only 12 (6.1\%) did not agree. When questioned regarding the best time to start SUP, 49 (24.9\%) said they would start SUP on arrival in hospital, 88 (44.7\%) would start only on ICU admission, and 60 (30.5\%) said they would start SUP only if there was a specific indication to do so (Fig. 2).

When asked about independent predictors of clinically important bleeding in ICU patients, 172 (87.3\%) predicted coagulopathy, 130 (65.9\%) predicted mechanical ventilation, 160

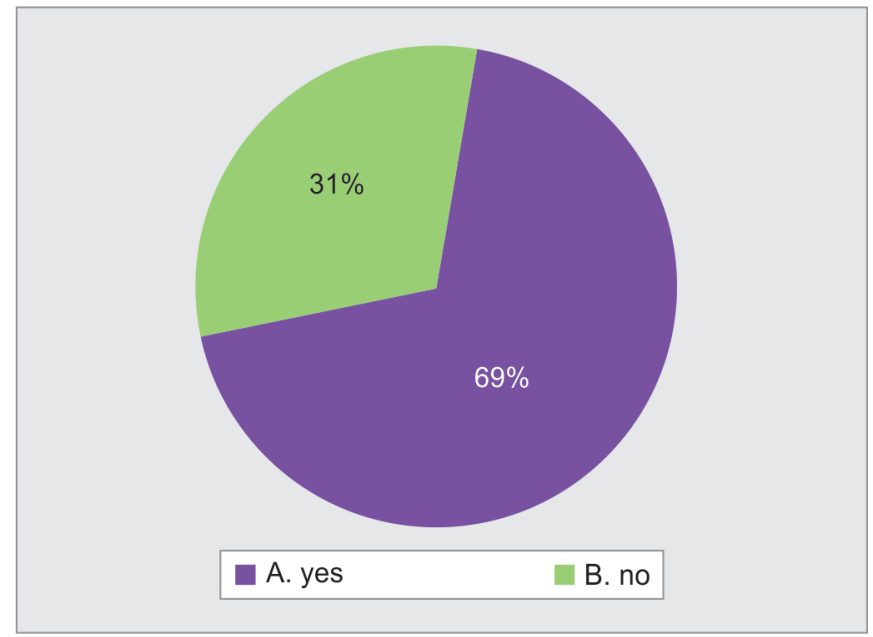

Fig. 1: Response to whether all patients should receive stress ulcer prophylaxis
(81.2\%) considered the use of nonsteroidal anti-inflammatory drugs (NSAIDs) and 36 (18.3\%) considered diabetes mellitus an independent major factor. Eighty-four respondents (42.6\%) would initiate SUP in ICUs only if at least one independent/major risk factor is present, 27 (13.7\%) when at least one minor risk factor is present, and 86 (43.7\%) even when no risk factors were involved. Fifty-nine (29.9\%) thought that one minor risk factor was enough to warrant the use of SUP, 87 (44.2\%) thought two minor risk factors were required, 37 (18.8\%) thought three were required, and very few thought that that four, five, or more risk factors were required for SUP prophylaxis.

One hundred and twenty respondents (60.9\%) said they would initiate SUP at the time of initiation of mechanical ventilation, 37 (18.8\%) would initiate after 24 hours, 39 (19.8\%) after 48 hours, and $1(0.5 \%)$ after 7 days of mechanical ventilation.

One hundred thirty-three (67.5\%) patients would start a PPI, $30(15.2 \%)$ an H2RA, and 34 (17.3\%) would start sucralfate in the presence of major risk factors. The PPI was considered the best SUP for preventing bleeding by 142 (72.1\%), H2RA by 19 (9.6\%), and sucralfate by 34 (17.3\%) respondents (Fig. 3). One hundred fourteen (58\%) respondents thought that PPIs were associated with the maximum risk of ventilator-associated pneumonia (VAP), while 41 (20.8\%) and 42 (21.3\%) believed H2RA and sucralfate, respectively, to be associated with the highest risk of VAP. One hundred and twenty-eight (65\%) were aware that VAP was an adverse effect of PPI therapy, 137 (69.5\%) believed that $C$. difficile infection may occur due to PPI therapy; and 31 (15.7\%) and 28 (14.2\%), respectively, thought that this therapy may lead to myocardial ischemia and dementia. One hundred and twenty-two respondents (61.9\%) were of the opinion that an increased risk of community as well as hospital-acquired pneumonia was observed in patients using PPIs. In the presence of minor risk factors, 75 (38.1\%) would use H2RA, 61 (31\%) would use PPI, and another 61 (31\%) would use sucralfate.

One hundred nineteen (60.4\%) preferred to use the enteral route over parenteral for giving SUP. Only 33\% knew that both parenteral and enteral routes have a similar efficacy for providing SUP.

When asked about the appropriate time to stop SUP, 69 (35\%) said they would continue throughout the patient's ICU stay, 42 (21.3\%) would continue throughout the patient's hospital stay, and $86(43.7 \%)$ respondents would stop it when there is no remaining indication.

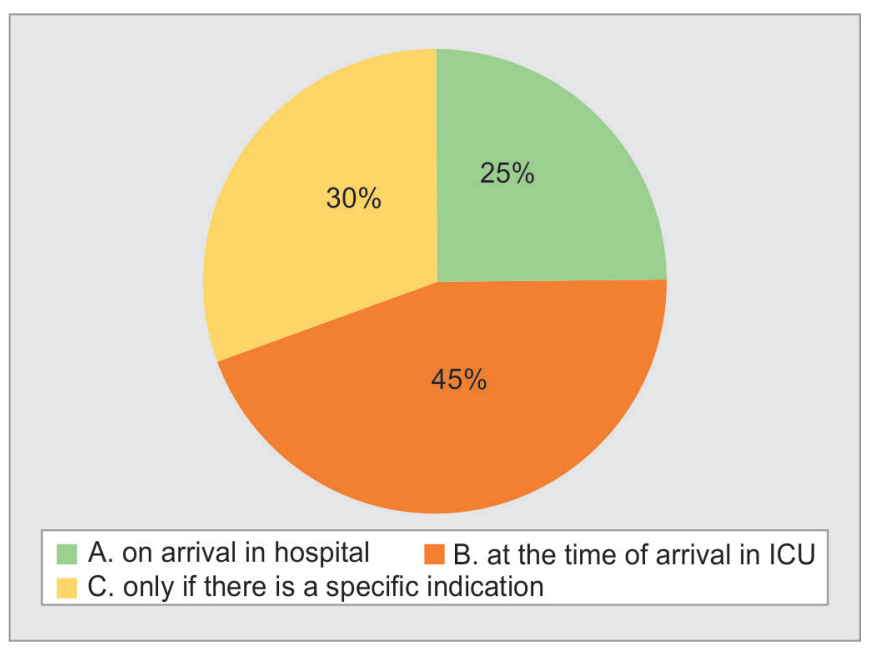

Fig. 2: Responses for correct time to start stress ulcer prophylaxis 


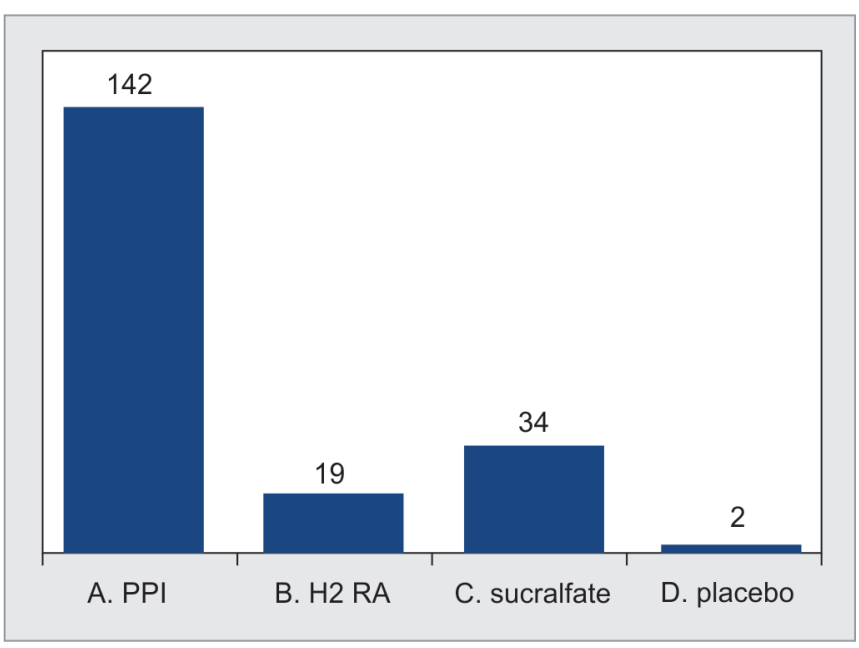

Fig. 3: Responses to the best stress ulcer prophylaxis drug

\section{Discussion}

For several decades, preventing stress ulcerations in ICU patients has prompted the extensive use of SUP. In 1994, Cook et al. conducted a landmark trial and identified the most significant risk factors for stress ulceration is mechanical ventilation for more than 48 hours and a primary coagulopathy $(p<0.001) .{ }^{2}$ However, since then, there has been a dramatic reduction in the incidence of clinically significant bleeding due to stress ulceration in hospitalized patients and an increase in recognition of the multiple side effects of the drugs used for SUP.

Stress ulcers are superficial lesions involving the mucosal layer of the stomach especially fundal region, which usually occurs following a major stress such as surgery, trauma, or organ failure when the mucosal barrier of the GI tract (GIT) is compromised and becomes susceptible to damage by hydrogen ions and free radicals. ${ }^{3,4}$ In a critical illness, the increased release of catecholamines and hypovolemia lead to a fall in the cardiac output, leading to vasoconstriction and release of proinflammatory cytokines and splanchnic hypoperfusion. This further leads to decreased secretion of bicarbonate, decreased mucosal blood flow and GIT motility, and acid back diffusion which renders the mucosa vulnerable to acid damage. ${ }^{4}$ While the causes of stress ulceration are multifactorial, the root cause is decreased blood flow in the gut microcirculation which in turn causes disruption of the gastric mucosal barrier and hypersecretion of acid. ${ }^{5}$ Also, there is a release of various mediators such as oxygen-free radicals and cytokines. ${ }^{5}$

In ICU patients, although the development of stress ulcer is quite high, the incidence of clinically significant Gl bleeding (CSGIB) is low. ${ }^{6}$ In ICU patients, the main causes of splanchnic hypoperfusion and mucosal ischemia are shock or changes in intrathoracic pressure, due to mechanical ventilation. ${ }^{4}$ Mechanical ventilation promotes renin angiotensin aldosterone system (RAAS) activity and catecholamine release, and the use of high positive end expiratory pressure (PEEP) may decrease venous return and reduce preload and cardiac output. Other factors predisposing to gastric ulceration are the use of medications such as opioids and sedatives that decrease gut motility and impair venous return, vasopressors for hemodynamic instability that cause splanchnic vasoconstriction, and glucocorticoids and NSAID therapy. In addition, the presence of a coagulopathy impairs the ability to terminate any active bleeding., 4
The questionnaire was sent to 550 clinicians. One hundred and thirty-two (67\%) of the respondents were aware of guidelines regarding SUP and $68 \%$ said that SUP protocols were being followed in their respective ICUs. However, in the absence of clear-cut guidelines in the literature, it becomes mandatory for physicians and intensivists to be cognizant of the actual incidence of serious bleeding in ICU patients and the current indications for SUP and the side effects of drugs used for the same.

Sixty-nine percent respondents opined that all patients in the ICU should receive SUP prophylaxis. Recent studies demonstrated the significant decline in the incidence of CSGIB. ${ }^{8}$ This may be attributed to improvement in the management of ICU patients. Early goal-directed therapy promotes prompt and aggressive fluid resuscitation in patients with sepsis, which may decrease gastric hypoperfusion and reduce stress ulcerations. ${ }^{9}$ Also, improvements in technological advances to assess fluid status and the use of specialized monitors to measure parameters such as stroke volume variation and pulse pressure variation have allowed a better optimization of fluid status. In addition, it has been learned that the use of lower tidal volumes and lower plateau pressures during mechanical ventilation improves the outcome in ICU patients receiving mechanical ventilation. ${ }^{10}$ Another major change in ICU practice over the past few decades which has lowered the risk of CSGIB has been the promotion of early enteral nutrition.

Only 64 respondents (32.5\%) were aware that bleeding from stress ulceration is extremely uncommon in intensive care patients, whereas $67.5 \%$ thought that it is common. Literature shows that although the stress-related mucosal disease is very common in ICU patients and may occur in almost $75-100 \%$ patients within the first 24 hours of admission, approximately $5-25 \%$ will have bleeding and only $1-4 \%$ will have clinically significant bleeding. Severe ulceration and bleeding may lengthen the duration of ICU stay by up to 8 days and increase mortality by as much as fourfold. ${ }^{6,11,12}$ Clinically significant $\mathrm{Gl}$ bleeding has been defined variously as bleeding requiring blood transfusion or a fall in hemoglobin of $2 \mathrm{~g} / \mathrm{dL}$ plus 2 units transfusion over 24 hours or hemodynamic compromise defined as a fall in systolic blood pressure of $20 \mathrm{~mm} \mathrm{Hg}$ within 24 hours or a $10 \mathrm{~mm}$ decrease with increase in heart rate of $20 \mathrm{bpm}$ on standing. ${ }^{6}$ Thirty-six percent respondents thought that the risk of bleeding may not be altered by the use of acid-suppressive therapy, whereas 126 (64\%) believed that acid-suppression drugs do alter the risk of bleeding. Krag et al. studied 1,034 patients in 97 ICUs, where $73 \%$ (71-76\%) of patients received acid suppressants; most received PPIs. Clinically important Gl bleeding occurred in $2.6 \%$ of patients and the use of acid suppressants (odds ratio 3.6, 1.3-10.2) was independently associated with it. In patients with CSGIB, crude and adjusted odds for mortality were 3.7 (1.7-8.0) and 1.7 (0.7-4.3), respectively. Surprisingly, the use of SUP on the first day of ICU stay was associated with an increased risk of Gl bleeding. ${ }^{13}$

One of the major factors that has reduced the incidence of CSGIB has been the emphasis on initiation of early enteral nutrition, and most respondents (93.9\%) were aware that enteral tube feeding initiated within 48 hours of ICU admission may be protective against stress ulceration. Nutrition provides fuel for metabolism, attenuates the catabolic stress response, and prevents cellular injury. Also, enteral nutrition may be protective against formation of stress ulcers by leading to an increase in the gastric $\mathrm{pH}$ and providing protection from cytotoxins. ${ }^{4,8,14}$ Tolerance of enteral nutrition in critically ill patients depends on adequate perfusion of the gut and suggests the absence of splanchnic ischemia. Palm et al. evaluated 
the incidence of CSGIB in intubated surgical/trauma ICU patients receiving enteral nutrition and found no benefit of pharmacologic SUP. ${ }^{15}$ Similarly, Selvanderan et al. found that prophylactic pantoprazole demonstrated no benefit to mechanically ventilated patients receiving enteral nutrition. ${ }^{16}$

When questioned regarding the initiation of SUP, only 60 respondents $(30.5 \%)$ replied that they would initiate SUP only in the presence of a specific indication. Prolonged mechanical ventilation, use of certain medications, and coexisting coagulopathy have been historically considered to be the associated factors that predispose to bleeding from stress ulceration. ${ }^{4,7}$ In 1999, the original guidelines for SUP, formulated by the AHSP, recommended SUP in patients with coagulopathy or patients requiring mechanical ventilation for more than 48 hours, history of Gl ulceration, or bleeding within the last 1 year prior to admission and in patients with two or more of the following risk factors: sepsis, ICU stay greater than 1 week, occult bleeding lasting at least 6 days, and use of high-dose corticosteroids (>250 mg/day of hydrocortisone or the equivalent). Prophylaxis was also recommended for ICU patients with a Glasgow coma score of $\leq 10$ (or the inability to obey simple commands), thermal injuries to $>35 \%$ of their body surface area (BSA), following partial hepatectomy, ICU patients with polytrauma (e.g., injury severity score of $\geq 16$ ), transplantation patients in the ICU perioperatively, and ICU patients with hepatic failure and with spinal cord injuries. ${ }^{1}$ They also delineated independent risk factors for Gl bleed in which treatment rather than prophylaxis with acid suppressing drugs is indicated. These included Zollinger-Ellison syndrome, acute upper GI bleed, erosive esophagitis, Helicobacter pylori treatment, gastric or duodenal ulcer, gastroesophageal reflux disease, and regular scheduled use of an acid-suppressing medication prior to admission. ${ }^{1}$

However, by the year 2000s, the scenario seemed to have changed. Quenot et al. evaluated the use of SUP in critically ill ICU patients and reiterated that the mechanism of stress ulceration is multifactorial and incompletely understood. ${ }^{17}$ The various drugs used for SUP have significant side effects which may outweigh their utility. Intravenous H2RAs raise gastric $\mathrm{pH}$ but may be associated with development of tolerance, various drug interactions, and neurologic manifestations. Sucralfate is administered by the nasogastric route to protect the gastric mucosa without raising $\mathrm{pH}$ but may decrease absorption of oral medications administered simultaneously. The PPIs are suggested to be the most potent pharmacologic agents for acid inhibition and are at least as effective as H2RAs but are associated with a myriad of side effects. Recently, Rafinazari et al. studied the use of SUP during ICU stay and found that $80 \%$ patients received SUP, in which $44.4 \%$ showed no indication according to the AHSP guidelines; while $6.3 \%$ did not receive SUP though it was indicated. ${ }^{18}$

In 2015, Krag et al. found that 73\% ICU patients received SUP and $59 \%$ patients with clinically important GI bleed had received SUP prior to bleed. ${ }^{13}$ Krag et al. found that CSGIB was associated with use of SUP on day 1 but not with mechanical ventilation. In the POP-UP study, Selvanderan et al. ${ }^{16}$ evaluated the use of daily intravenous (IV) placebo or pantoprazole in mechanically ventilated ICU patients suitable for enteral nutrition and found no evidence of either benefit or harm with prophylactic administration of pantoprazole. Similarly, a more recent placebo-controlled trial ( $n=102)$ found no difference in bleeding complications when pantoprazole or placebo was used in addition to enteral nutrition in mechanically ventilated patients. ${ }^{19}$ Alhazzani et al. in the REVISE pilot trial have demonstrated the feasibility of a randomized, international, double-blinded, multicenter trial to investigate the effect of pantoprazole vs placebo in mechanically ventilated patients. ${ }^{20}$ Hospital or overall mortality did not show significant differences in the two groups, and there was a trend toward reduced infectious complications in placebo-treated patients, suggesting that it may be possible to withdraw the use of pantoprazole from ICU patient altogether as it does not seem to increase the risks of GI bleeding or mortality. The SUP-ICU trial by Krag et al. which is an international, multicenter, randomized, blinded, parallel-group trial of SUP with a PPI vs placebo (saline) is expected to provide high-quality data regarding the pros and cons of SUP with a PPI in critically ill adult patients in the ICU. ${ }^{21}$

One hundred and thirty-three (67.5\%) respondents' choice of drug for SUP was a PPI, 15.2\% preferred the use of an H2RA, and $17.3 \%$ would start sucralfate. A PPI was considered the best SUP for preventing bleeding by 142 respondents (72.1\%), H2RA by $19(9.6 \%)$ and sucralfate by $34(17.3 \%)$ respondents. According to the ASHP guidelines, the drug of choice among antacids, $\mathrm{H} 2$-receptor blockers, and sucralfate for use, as SUP should be made on an institutional basis, taking into consideration the route of administration (is enteral possible), potential side effects, and the cost factor. ${ }^{1}$ When considering the choice of drug, in various metaanalyzes, the use of PPI for SUP has been found to be associated with a significantly lower rate of CSGIB compared to an H2RA. ${ }^{22-24}$

The main concern associated with use of a PPI in the ICU is the higher risk of infections, particularly, pneumonia and $C$. difficile probably due to the attenuation of gastric acid protection against bacteria. ${ }^{25}$

Alshamsi et al. conducted a systemic review and meta-analysis of 19 trials enrolling 2,117 patients. The PPIs were found to be superior to H2RAs in preventing clinically important and overt GI bleeding, without significantly increasing the risk of pneumonia or mortality. However, their impact on C. difficile infection remained undetermined. ${ }^{23}$ More recently, Alhazzani and colleagues included 57 trials enrolling 7,293 patients of 96 potentially eligible studies. Their results suggested that PPIs are probably more effective in preventing clinically important Gl bleeding (CIB) when compared to H2RA, sucralfate, and a placebo. There were no significant differences between H2RA, sucralfate, and placebo. The PPIs probably increased the risk of developing pneumonia when compared to H2RAs, sucralfate, and placebo. Their results also indicated that mortality is probably similar across interventions. The estimates of risks of bleeding were found to vary significantly across different studies. Only one study reported on $C$. difficile infection. Also, it was found that the definitions of pneumonia varied considerably in different trials, and most studies on sucralfate predated pneumonia prevention strategies. They concluded that there was only moderate quality evidence to indicate that PPIs are the most effective agents in preventing $\mathrm{CIB}$, but they may increase the risk of pneumonia and opined that the risks and advantages of routine use of SUP still remain open to debate. ${ }^{26}$

On asking about the discontinuation of SUP, respondents had mixed opinion. However, as early as 1999, the AHSP guidelines do not support SUP for adult patients in non-ICU settings. ${ }^{1}$ Rafinazari et al. also reported that, in their study, $38.5 \%$ of patients did not receive appropriate SUP on ICU admission and $81.2 \%$ continued on inappropriate SUP upon transfer from ICU. ${ }^{18}$ Wohlt et al. reported that 394 patients met the eligibility criteria for SUP, 357 patients were prescribed SUP, and $80 \%$ continued SUP on transfer from ICU. ${ }^{27}$ 
This also imposes an unnecessary financial burden and increases the cost of healthcare.

According to the ASHP guidelines, ${ }^{1}$ regarding the route of SUP administration, sucralfate should be preferred if the patient has gastric access. If there is no gastric access but there is enteral access, then oral $\mathrm{H} 2$ blocker can be used; and if there is no enteral access at all, an IV H2 blocker should be used. It was only after this that PPIs come into routine use and may be preferred if at all the patient warrants SUP.

Administration of PPIs is not without certain definite risks, and only recently the medical fraternity is appreciating the changes that they provoke in the intestinal microbial environment and other anti-infective defense mechanisms of the body. ${ }^{28}$ Thus, there is an ambivalence in the literature with regard to SUP in ICU patients; and new guidelines are still awaited, the only available guidelines being of 1999, i.e., 31 years old. The literature seems to suggest that in those patients receiving and tolerating enteral nutrition, it is unlikely that there is a state of gastric hypoperfusion and increased risk of CSGIB, and hence SUP may not be required or beneficial. ${ }^{29}$ On the contrary, SUP may be indicated in patients not allowed or not tolerating enteral nutrition if historically accepted high-risk conditions coexist for the development of stress ulcerations such as prolonged mechanical ventilation, coagulopathy, high degree burns, etc. If available, the gastric/enteral route may be preferred to the IV route. The PPIs seem to have better efficacy for preventing CSGIB and may be preferred if the IV route is used although the complications associated with their use may be higher as compared to $\mathrm{H} 2$ blockers. Also, once initiated, SUP must be discontinued as soon as the indication for its initiation no longer exists. The results of our survey indicate that there is a lack of clarity regarding SUP in ICU patients. Stress ulcer prophylaxis is not indicated in all ICU patients and the decision to institute SUP must be individualized in each patient. However, most importantly, institutional protocols for SUP must be in place which should clearly define the indications, route, timings of initiation, discontinuation, and the availability and cost implications of the drugs used for SUP.

\section{References}

1. ASHP Commission on Therapeutics. ASHP therapeutic guidelines on stress ulcer prophylaxis. Am J Health-Syst Pharm 1999;56(4):347-379. DOI: 10.1093/ajhp/56.4.347.

2. Cook DJ, Fuller HD, Guyatt GH, Marshall JC, Leasa D, Hall R, et al. Risk factors for gastrointestinal bleeding in critically ill patients. Canadian critical care trials group. N Engl J Med 1994;330(6):377-381. DOI: 10.1056/NEJM199402103300601.

3. Barletta JF, Mangram AJ, Sucher JF, Zach V. Stress ulcer prophylaxis in neurocritical care. Neurocrit Care 2018;29(3):344-357. DOI: 10.1007/ s12028-017-0447-y.

4. Stollman N, Metz DC. Pathophysiology and prophylaxis of stress ulcer in intensive care unit patients. J Crit Care 2005;20(1):35-45. DOI: 10.1016/j.jcrc.2004.10.003.

5. Bardou M, Quenot JP, Barkun A. Stress-related mucosal disease in the critically ill patient. Nat Rev Gastroenterol Hepatol 2015;12(2):98-107. DOI: 10.1038/nrgastro.2014.235.

6. Fennerty MB. Pathophysiology of the upper gastrointestinal tract in the critically ill patient: rationale for the therapeutic benefits of acid suppression. Crit Care Med 2002;30(Supplement):S351-S355. DOI: 10.1097/00003246-200206001-00002.

7. Buendgens $L$, Koch A, Tacke F. Prevention of stress-related ulcer bleeding at the intensive care unit: risks and benefits of stress ulcer prophylaxis. World J Crit Care Med 2016;5(1):57-64. DOI: 10.5492/ wjccm.v5.i1.57.
8. Plummer MP, Blaser A, Deane A. Stress ulceration: prevalence, pathology and association with adverse outcomes. Crit Care 2014;18(2):213-219. DOI: 10.1186/cc13780.

9. Dellinger RP, Levy MM, Rhodes A, Annane D, Gerlach H, Opal $S M$, et al. Surviving sepsis campaign: international guidelines for management of severe sepsis and septic shock: 2012. Crit Care Med 2013;41(2):580-637. DOI: 10.1097/CCM.0b013e31827e83af.

10. Acute Respiratory Distress Syndrome Network, Brower RG, Matthay MA, Morris A, Schoenfeld D, Thompson BT, et al. Ventilation with lower tidal volumes as compared with traditional tidal volumes for acute lung injury and the acute respiratory distress syndrome. $N$ Engl J Med 2000;342(18):1301-1308. DOI: 10.1056/NEJM200005043421801.

11. Cook DJ, Griffith LE, Walter SD, Guyatt GH, Meade MO, Heyland DK, et al. The attributable mortality and length of intensive care unit stay of clinically important gastrointestinal bleeding in critically ill patients. Crit Care 2001;5(6):368-375. DOI: 10.1186/cc1071.

12. Laine L, Takeuchi K, Tarnawski A. Gastric mucosal defense and cytoprotection: bench to bedside. Gastroenterology 2008;135(1): 41-60. DOI: 10.1053/j.gastro.2008.05.030.

13. Krag M, Perner A, Wetterslev J, Wise MP, Borthwick M, Bendel S, et al. Prevalence and outcome of gastrointestinal bleeding and use of acid suppressants in acutely ill adult intensive care patients. Intensive Care Med 2015;41(5):833-845. DOI: 10.1007/s00134-015-3725-1.

14. Mohebbi L, Hesch K. Stress ulcer prophylaxis in the intensive care unit. Proc (Bayl Univ Med Cent) 2009;22(4):373-376. DOI: 10.1080/08998280.2009.11928562.

15. Palm NM, McKinzie B, Ferguson PL, Chapman E, Dorlon M, Eriksson EA, et al. Pharmacologic stress gastropathy prophylaxis may not be necessary in at-risk surgical trauma ICU patients tolerating enteral nutrition. J Intensive Care Med 2018;33(7):424-429. DOI: 10.1177/0885066616678385

16. Selvanderan SP, Summers MJ, Finnis ME, Plummer MP, Ali Abdelhamid $Y$, Anderson MB, et al. Pantoprazole or placebo for stress ulcer prophylaxis (POP-UP): randomized double-blind Exploratory study. Crit Care Med 2016;44(10):1842-1850. DOI: 10.1097/ CCM.0000000000001819.

17. Quenot JP, Thiery N, Barbar S. When should stress ulcer prophylaxis be used in the ICU? Curr Opin Crit Care 2009;15(2):139-143. DOI: 10.1097/ MCC.0b013e32832978e0.

18. Rafinazari N, Abbasi S, Farsaei S, Mansourian M, Adibi P. Adherence to stress-related mucosal damage prophylaxis guideline in patients admitted to the intensive care unit. J Res Pharm Pract 2016;5(3): 186-192. DOI: 10.4103/2279-042X.185728.

19. El-Kersh K, Jalil B, McClave SA, Cavallazzi R, Guardiola J, Guilkey K, et al. Enteral nutrition as stress ulcer prophylaxis in critically ill patients: a randomized controlled exploratory study. J Crit Care 2018;43:108-113. DOI: 10.1016/j.jcrc.2017.08.036.

20. Alhazzani W, Guyatt G, Alshahrani M, Deane AM, Marshall JC, Hall $\mathrm{R}$, et al. Withholding pantoprazole for stress ulcer prophylaxis in critically ill patients: a pilot randomized clinical trial and meta-analysis. Crit Care Med 2017;45(7):1121-1129. DOI: 10.1097/ CCM.0000000000002461.

21. Krag M, Perner A, Wetterslev J, Wise MP, Borthwick M, Bendel S, et al. Stress ulcer prophylaxis with a proton pump inhibitor vs placebo in critically ill patients (SUP-ICU trial): study protocol for a randomised controlled trial. Trials 2016;17(1):205. DOI: 10.1186/s13063-0161331-3.

22. Pongprasobchai S, Kridkratoke S, Nopmaneejumruslers C. Proton pump inhibitors for the prevention of stress-related mucosal disease in critically-ill patients: a meta-analysis. J Med Assoc Thai 2009:92:632-637.

23. Alshamsi F, Belley-Cote E, Cook D, Almenawer SA, Alqahtani Z, Perri $D$, et al. Efficacy and safety of proton pump inhibitors for stress ulcer prophylaxis in critically ill patients: a systematic review and metaanalysis of randomized trials. Crit Care 2016;20(1):120. DOI: 10.1186/ s13054-016-1305-6.

24. Alhazzani W, Alenezi F, Jaeschke RZ, Moayyedi P, Cook DJ. Proton pump inhibitors vs histamine 2 receptor antagonists for stress 
ulcer prophylaxis in critically ill patients: a systematic review and meta-analysis. Crit Care Med 2013;41(3):693-705. DOI: 10.1097/ CCM.0b013e3182758734.

25. MacLaren R, Reynolds PM, Allen RR. Histamine-2 receptor antagonists vs proton pump inhibitors on gastrointestinal tract hemorrhage and infectious complications in the intensive care unit. JAMA Intern Med 2014;174(4):564-574. DOI: 10.1001/jamainternmed.2013. 14673.

26. Alhazzani W, Alshamsi F, Belley-Cote E, et al. Efficacy and safety of stress ulcer prophylaxis in critically ill patients: a network meta-analysis of randomized trials. Intensive Care Med 2018;44(1):1-11. DOI: 10.1007/ s00134-017-5005-8.

27. Wohlt P, Hansen LA, Fish JT. Inappropriate continuation of stress ulcer prophylactic therapy after discharge. Ann Pharmacother 2007;41(10):1611-1616. DOI: 10.1345/aph.1K227.

28. Buendgens $L$, Tacke F. Do we still need pharmacological stress ulcer prophylaxis at the ICU? J Thorac Dis 2017;9(11):4201-4204. DOI: 10.21037/jtd.2017.09.121.

29. Stepanski M, Palm N. We no longer need to stress ulcer prophylaxis in the critically ill. J Intensive \& Crit Care 2017;3(02):2. DOI: 10.21767/24718505.100086. 
Appendix 1: Questionnaire with results

1. Which department do you work in?
A. Anesthesiology
B. Medicine
C. Intensivists

2. What is your place of work?
A. Government medical college
B. Government hospital
C. Private medical college
D. Corporate hospital
E. Private nursing home

3. Which city do you work in?
A. Delhi
B. Metro other than Delhi
C. Others

4. How many years of experience do you have?
A. $0-3$ years
B. 3-6 years
C. 6-10 years
D. $>10$ years

5. What is the duration of ICU experience?
A. $<6$ months
B. 6-12 months
C. $1-5$ years
D. $>5$ years

6. Do you know of any guidelines regarding stress ulcer prophylaxis?

A. Yes

B. No

7. Is any SUP protocol being followed in your ICU?
A. Yes
B. No

8. Should all patients in ICU receive SUP?

A. Yes

B. No

9. Is bleeding from stress ulceration extremely uncommon in ICU patients?
A. Yes
B. No

10. The risk of bleeding may not be altered by the use of acid suppressive therapy:

A. Yes

B. No

11. Early enteral tube feeding (initiated within 48 hours of ICU admission) may be protective against stress ulceration

A. Yes

B. No

12. When is the best time to start SUP?

A. On arrival in hospital

B. At the time of arrival in ICU

C. Only if there is a specific indication

13. Only patients with risk factors for stress related mucosal diseases should receive SUP?
A. Yes
B. No

14. Independent predictors of clinically important bleeding in ICU pts are (tick the correct options; may be $>1$ )

A. Coagulopathy

B. NSAIDS

C. DM

D. Mechanical ventilation $>48$ hours

\begin{tabular}{|c|c|}
\hline 180 & 91.4 \\
\hline 11 & 5.6 \\
\hline 6 & 3 \\
\hline 101 & 51.3 \\
\hline 32 & 16.2 \\
\hline 13 & 6.6 \\
\hline 45 & 22.8 \\
\hline 6 & 3 \\
\hline 136 & 69 \\
\hline 16 & 8.1 \\
\hline 45 & 22.8 \\
\hline 71 & 36 \\
\hline 72 & 36.5 \\
\hline 15 & 7.6 \\
\hline 39 & 19.8 \\
\hline 58 & 29.4 \\
\hline 49 & 24.9 \\
\hline 57 & 28.9 \\
\hline 33 & 16.8 \\
\hline 132 & 67 \\
\hline 65 & 33 \\
\hline 134 & 68 \\
\hline 63 & 32 \\
\hline 136 & 69 \\
\hline 61 & 31 \\
\hline 64 & 32.5 \\
\hline 133 & 67.5 \\
\hline 71 & 36 \\
\hline 126 & 64 \\
\hline 185 & 93.9 \\
\hline 12 & 6.1 \\
\hline 49 & 24.9 \\
\hline 88 & 44.7 \\
\hline 60 & 30.5 \\
\hline 77 & 39.1 \\
\hline 120 & 60.9 \\
\hline
\end{tabular}

$172 \quad 87.3$

$160 \quad 81.2$

$36 \quad 18.3$

$130 \quad 66$

1.4 5.6 
Contd...

Question

Responses Responses (\%)

15. When do you consider initiating SUP in mechanically ventilated patients?
A. At the time of initiation of ventilation
B. Mechanical ventilation $>24$ hours
C. Mechanical ventilation $>48$ hours
D. Mechanical ventilation $>7$ days

120

60.9

18.8

19.8

$\begin{array}{rr}39 & 19.8 \\ 1 & 0.5\end{array}$

16. SUP should be initiated in the ICU setting if:

A. At least 1 independent/major risk factor is present $\quad 84 \quad 42.6$

B. When at least 1 minor risk factor is present $\quad 27 \quad 13.7$

C. Even when there are no risk factors

$86 \quad 43.7$

17. Which is the SUP of choice in presence of major risk factors?
A. PPI
B. H2 RA
C. Sucralfate
A. PPI
B. H2 RA
C. Sucralfate
D. Placebo

$133 \quad 67.5$

$30 \quad 15.2$

$34 \quad 17.3$

18. Which is the best SUP to prevent gastric bleed?

19. Which SUP has maximum risk of ventilator associated pneumonia?
A. PPI
B. H2 RA
C. Sucralfate

$142 \quad 72.1$

$19 \quad 9.6$

$34 \quad 17.3$

$2 \quad 1$

20. Adverse effects of PPIs include (tick $\geq 1$ correct answer)

A. VAP

B. Clostridium difficile infection

C. MI

D. Dementia

21. In patients using PPIs, there is an increased risk of community as well as hospital-acquired pneumonia.
A. Yes

B. No

22. Presence of how many minor risk factors warrants use of SUP prophylaxis?
A. 1
B. 2
C. 3
D. 4
E. $\geq 5$

$\begin{array}{rc}59 & 29.9 \\ 87 & 44.2 \\ 37 & 18.8 \\ 8 & 4.1 \\ 6 & 3 \\ & \\ 61 & 31 \\ 75 & 38.1 \\ 61 & 31 \\ & \\ 78 & 39.6 \\ 119 & 60.4 \\ & \\ 65 & 33 \\ 132 & 67 \\ & \\ 69 & 35 \\ 42 & 21.3 \\ 0 & 0 \\ 86 & 43.7\end{array}$

